\title{
Strates
}

STRATES Matériaux pour la recherche en sciences sociales

Hors-série | 2002

Parcours dans la recherche urbaine, Michel Rochefort, un géographe engagé

\section{La formation des urbanistes en question}

Jacques Brun et Catherine Paix

\section{(2) OpenEdition}

Journals

Édition électronique

URL : http://journals.openedition.org/strates/573

DOI : $10.4000 /$ strates. 573

ISSN : $1777-5442$

Éditeur

Laboratoire Ladyss

Édition imprimée

Date de publication : 1 janvier 2002

ISSN : 0768-8067

Référence électronique

Jacques Brun et Catherine Paix, "La formation des urbanistes en question », Strates [En ligne], Hors-

série | 2002, mis en ligne le 18 mai 2005, consulté le 08 septembre 2020. URL : http://

journals.openedition.org/strates/573; DOI : https://doi.org/10.4000/strates.573

Ce document a été généré automatiquement le 8 septembre 2020

Tous droits réservés 


\title{
La formation des urbanistes en question
}

\author{
Jacques Brun et Catherine Paix
}

1 Tu es président de l'Institut français d'urbanisme, tu interviens également dans la formation des urbanistes en Tunisie; comment envisages-tu la formation des urbanistes aujourd'hui ?Quelles devraient en être les lignes directrices? Tu nous a dit : «Est-ce que l'urbanisme existe encore de nos jours?» Pourquoi une telle interrogation?

2 Je pense qu'il est nécessaire de donner aux urbanistes les moyens de comprendre la réalité urbaine dans sa dynamique globale. Il faut que tous ceux qui vont s'occuper d'aménagement urbain aient cette connaissance préalable, sinon ils ne vont faire que de la technocratie. Mais il y a une spécialisation de plus en plus poussée des métiers de la ville qui demande de repenser nos enseignements. Nous sommes dans une société qui n'est plus du tout apte à poser les problèmes de la ville dans leur globalité, parce qu'il y a trop de forces - qui sont des forces néo-libérales - que l'on ne maitrise pas, et parce que les urbanistes ont à aborder des problèmes d'ordre très différent qui exigent une spécialisation. On ne peut pas en rester à une conception de l'urbanisme avec un grand «U » qui permettrait d'embrasser tous les problèmes de la ville dans leur ensemble. Il faut donc assurer à chacun une connaissance globale de la ville et une formation à un métier, sinon nos instituts vont fabriquer des chômeurs. Les employeurs qui sont venus à la table ronde organisée récemment à l'IFU l'ont exprimé très clairement. Pour eux l'emploi, c'est avant tout un métier. Ils reconnaissent que les urbanistes doivent avoir une formation générale mais pour eux, ils doivent également être spécialisés dans un domaine précis.

3 Si l'on était dans une société où l'on puisse véritablement contrecarrer le jeu des forces économiques, on pourrait essayer de repenser la ville dans sa globalité. Mais pour l'instant repenser la ville, c'est un exercice de style. Toujours à cette table ronde de l'IFU un Anglais, qui enseigne depuis 26 ans l'aménagement urbain, a montré comment la politique de privatisation de Margaret Thatcher avait détruit le Town-planning en Grande-Bretagne. Toutes les écoles d'urbanisme anglaises sont en train de fermer, même la grande école de Nottingham qui a été la plus prestigieuse dans ce domaine. 
Pour sauver nos instituts d'urbanisme il faut donc garder à l'esprit l'idée qu'il y a une formation générale indispensable à donner mais qu'en plus il faut être capable d'avoir plusieurs DESS qui préparent à des métiers de la ville. Si les instituts d'urbanisme tiennent à ne fabriquer que des gens qui vont enseigner et continuer à enseigner l'urbanisme en circuit fermé, on va avoir du chômage parmi les étudiants diplômés et on ne pourra pas continuer.

Est-ce qu'il n'y a pas non plus une tendance de la recherche à aller vers la segmentation?

5 Bien sûr, mais il s'agit dans ce cas d'une spécialisation qui se situe beaucoup plus au niveau de l'approfondissement des facteurs d'explication qu'au niveau de techniques permettant une application. Il ne faut quand même pas oublier que la recherche pose toujours des problèmes plus généraux même si la complexité conduit à la segmentation. Dans tous les domaines, on est amenés à spécifier certaines lignes d'explications qu'on approfondit, mais on le fait sans oublier l'interaction avec le reste. Le problème se pose différemment quand il s'agit de former des urbanistes, c'est-à-dire des gens qui vont agir, et dont le métier sera d'appliquer. Il s'agit de spécialisations beaucoup plus terre à terre, beaucoup plus techniques, qui demandent d'avoir une culture générale mais pour traiter de problèmes qui nécessitent une formation d'ingénieur. Il y a donc un tournant à prendre. Dans le milieu, les positions sur la question sont encore très partagées. Il y a ceux qui veulent maintenir l'idée que l'urbanisme c'est l'urbanisme avec un grand « $U$ » et d'autres, plus jeunes, qui voient que l'on est en train de fabriquer des chômeurs, ou que l'on a de moins en moins d'étudiants, ce qui est la même chose. Il y a matière à réflexion dans l'expérience anglaise. Contrairement à ce qu'il en est en Grande-Bretagne, où tout est bloqué, nous sommes en France dans une société où il y a encore une marge d'intervention de l'État et des collectivités locales dans l'aménagement urbain et où il $\mathrm{y}$ a donc une place pour des gens qui se destinent aux métiers de la ville. Il faut donc prendre un tournant, mais cela suppose de faire éclater la notion d'urbanisme.

\section{AUTEURS}

JACQUES BRUN

professeur à l'université de Paris I, Ladyss

CATHERINE PAIX

Ladyss, Cnrs 